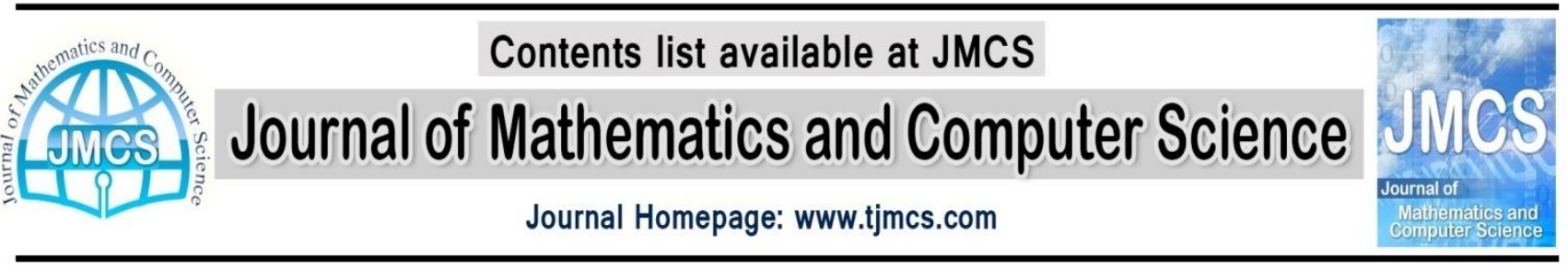

\title{
Some Refinements of Jensen's Inequality on Product Spaces
}

Article history:

\section{Peter O. Olanipekun ${ }^{1, *}$, Adesanmi A. Mogbademu1,+}

${ }^{1}$ Research Group in Mathematics and Applications Department of Mathematics, University of Lagos, Nigeria *peter.olanipekun1@students.unilag.edu.ng, +amogbademu@unilag.edu.ng

Received March 2015

Accepted July 2015

Available online July 2015

\section{Abstract}

In this paper, we give some refinements of the classical Jensen's inequality which generalizes some results already obtained in literatures.

Keywords: Convex function, Jensen's inequality, Fubini's theorem, $L^{p}$ spaces.

\section{Introduction}

In [3] J. Rooin refined the classical Jensen's inequality as

$$
\varphi\left(\int_{X} f d \mu\right) \leq \int_{Y} \varphi\left(\int_{X} f(x) \omega(x, y) d \mu(x)\right) d \lambda(y) \leq \int_{X}(\varphi \circ f) d \mu,
$$

where $(X, A, \mu)$ and $(Y, B, \lambda)$ are two probability measure spaces, $\omega: X \times Y \rightarrow[0, \infty)$ is a weight function on $X \times Y, I$ is an interval of the real line, $f \in L^{1}(\mu), f(x) \in I$ for all $x \in X$ and $\varphi$ is a real-valued convex function on $I$.

Also, in [2], the authors proved a generalization of the classical Jensen's inequality by Riemann-Stieltjes integration for two convex functions defined on an interval of $\mathbb{R}$.

In this paper, we generalize the above papers to a very general case by considering a more general abstract space i.e the $L^{p}$ spaces and two fuctions in this space. 


\section{Main Results}

We refine the classical Jensen's inequality on the $L^{p}$ spaces and show how our result generalizes those in literature.

Theorem 2.1. Let $\mathrm{X}$ be a measure space, with measure $\mu$. Let $f \in L^{p}(\mu)$ and $g \in L^{q}(\mu)$. Suppose $\varphi$ is any convex function and $\frac{1}{p}+\frac{1}{q}=1$, where $1<p<\infty$ and $1<q<\infty$ then the following inequality holds

$$
\varphi\left(\int_{X} f g d x\right) \leq\left(\int_{X} \varphi \circ f^{p} d x\right)^{\frac{1}{p}}\left(\int_{X} \varphi \circ g^{q} d x\right)^{\frac{1}{q}}
$$

\section{Proof:}

Let

$$
A=\left(\int_{X} \varphi \circ f^{p} d x\right)^{\frac{1}{p}}, \quad B=\left(\int_{X} \varphi \circ g^{q} d x\right)^{\frac{1}{q}}
$$

The case when $A=0$ is trivial. Also $A>0$ and $\mathrm{B}=\infty$ is trivial. So we consider the case $0<A<\infty, 0<$ $B<\infty$. We set

$$
F=\frac{\varphi \circ f}{A}, \quad G=\frac{\varphi \circ g}{B}
$$

Now,

$$
\int_{X} F^{p} d x=\int_{X} \frac{\varphi \circ f^{p} d x}{\int_{X} \varphi \circ f^{p} d x}=\int_{X} \frac{\varphi \circ g^{q} d x}{\int_{X} \varphi \circ g^{q} d x}=\int_{X} G^{q} d x=1
$$

Let $x \in X \ni 0<F(x)<\infty$ and $0<G(x)<\infty$ implies that $\exists s, t \in R \ni F(x)=e^{\frac{s}{p}}, \quad G(x)=e^{\frac{t}{q}}$. This implies

$$
\begin{array}{r}
e^{\frac{s}{p}+\frac{t}{q}} \leq p^{-1} e^{s}+q^{-1} e^{t} \\
F(x) G(x) \leq p^{-1} F^{p}(x)+q^{-1} G^{q}(x) \quad \forall x \in X
\end{array}
$$

Integrating both sides of (2.2), to obtain

$$
\int_{X} \frac{\varphi \circ f}{\left(\int_{X} \varphi \circ f^{p} d x\right)^{\frac{1}{p}}} \frac{\varphi \circ g}{\left(\int_{X} \varphi \circ g^{q} d x\right)^{\frac{1}{q}}} d x \leq 1 .
$$

This implies 


$$
\int_{X} \varphi \circ f \quad \varphi \circ g d x \leq\left(\int_{X} \varphi \circ f^{p} d x\right)^{\frac{1}{p}}\left(\int_{X} \varphi \circ g^{q} d x\right)^{\frac{1}{q}} .
$$

That is

$$
\begin{gathered}
\int_{X} \varphi(f g) d x \leq \int_{X} \varphi \circ f \quad \varphi \circ g d x \leq\left(\int_{X} \varphi \circ f^{p} d x\right)^{\frac{1}{p}}\left(\int_{X} \varphi \circ g^{q} d x\right)^{\frac{1}{q}} . \\
\varphi\left(\int_{X}(f g) d x\right) \leq \int_{X} \varphi(f g) d x \leq \int_{X} \varphi \circ f \varphi \circ g d x \leq\left(\int_{X} \varphi \circ f^{p} d x\right)^{\frac{1}{p}}\left(\int_{X} \varphi \circ g^{q} d x\right)^{\frac{1}{q}} .
\end{gathered}
$$

Remark 2.2. If $\varphi$ is an identity function then Theorem 2.1 gives Theorem 3.5 in [4]. For simplicity, we state it as Corollary 2.3.

Corollary 2.3. Let $\mathrm{X}$ be a measurable space, with measure $\mu$. Let $f$ and $g$ be measurable functions on $\mathrm{X}$ with range $[0, \infty]$. Suppose $\varphi$ is any identity function and $\frac{1}{p}+\frac{1}{q}=1$, where $1<p, q<\infty$. Then the following inequality holds

$$
\left(\int_{X} f g d x\right) \leq\left(\int_{X} f^{p} d x\right)^{\frac{1}{p}}\left(\int_{X} g^{q} d x\right)^{\frac{1}{q}}
$$

Theorem 2.4. Let $(X, A, \mu)$ and $(Y, B, \lambda)$ be two measure spaces and $\omega: X \times Y \rightarrow[0, \infty)$ be a weight function on $X \times Y$ such that

$$
\begin{aligned}
& \int_{X} \omega(x, y) d \mu(x)=1 \quad \forall \quad y \in Y, \\
& \int_{Y} \omega(x, y) d \lambda(y)=1 \quad \forall \quad x \in X .
\end{aligned}
$$

If $I$ is a measurable space, $f, g \in L^{p}(\mu), f(x) \in I \quad \forall \quad x \in X$ and $\varphi$ is a convex function in $I$, then

$$
\begin{aligned}
\varphi\left(\int_{X} f g d \mu\right) & \leq\left[\int_{Y} \varphi\left(\int_{X} f^{p}(x) \omega(x, y) d \mu(x)\right) d \lambda(y)\right]^{\frac{1}{p}} \\
& \times\left[\int_{Y} \varphi\left(\int_{X} g^{q}(x) \omega(x, y) d \mu(x)\right) d \lambda(y)\right]^{\frac{1}{q}} \\
& \leq\left[\int_{X} \varphi \circ f^{p} d \mu\right]^{\frac{1}{p}}\left[\int_{X} \varphi \circ g^{q} d \mu\right]^{\frac{1}{q}}
\end{aligned}
$$




\section{Proof:}

The functions $\omega$ and $(x, y) \rightarrow f(x)$ and so $(x, y) \rightarrow f^{p}(x) \omega(x, y)$ is product-measurable on $X \times Y$. The same thing goes for $g(x)$. We prove the first inequality. Clearly,

$$
\begin{aligned}
\left(\int_{X} \int_{Y}|f(x)|^{p} \omega(x, y) d \lambda(y) d \mu(x)\right)^{\frac{1}{p}} & =\left(\int_{X}|f(x)|^{p}\left(\int_{Y} \omega(x, y) d \lambda(y)\right) d \mu(x)\right)^{\frac{1}{p}} \\
& =\left(\int_{X}|f(x)|^{p} d \mu(x)\right)^{\frac{1}{p}} \\
& =\|f\|_{L^{p}(\mu)} \leq \infty .
\end{aligned}
$$

Similarly for $g(x)$, we have

$$
\begin{aligned}
\left(\int_{X} \int_{Y}|g(x)|^{q} \omega(x, y) d \lambda(y) d \mu(x)\right)^{\frac{1}{q}} & =\left(\int_{X}|g(x)|^{q}\left(\int_{Y} \omega(x, y) d \lambda(y)\right) d \mu(x)\right)^{\frac{1}{q}} \\
& =\left(\int_{X}|g(x)|^{q} d \mu(x)\right)^{\frac{1}{q}} \\
& =\|g\|_{L^{p}(\mu)} \leq \infty
\end{aligned}
$$

By Fubini's theorem we know that $(x, y) \rightarrow f^{p}(x) \omega(x, y)$ on $X \times Y$ belongs to $L^{p}(\mu \times \lambda)$. By the same argument, $(x, y) \rightarrow g^{q}(x) \omega(x, y)$ belongs to $L^{p}(\mu \times \lambda)$. Next, we define $F: Y \rightarrow R$ and $G: Y \rightarrow R$ by

$$
\begin{aligned}
& F(y)=\left(\int_{X} f^{p}(x) \omega(x, y) d \mu(x)\right)^{\frac{1}{p}}, \\
& G(y)=\left(\int_{X} g^{q}(x) \omega(x, y) d \mu(x)\right)^{\frac{1}{q}} .
\end{aligned}
$$

Now,

$$
\left[\int_{Y} \varphi\left(\int_{X} f^{p}(x) \omega(x, y) d \mu(x)\right) d \lambda(y)\right]^{\frac{1}{p}}\left[\int_{Y} \varphi\left(\int_{X} g^{q}(x) \omega(x, y) d \mu(x)\right) d \lambda(y)\right]^{\frac{1}{q}}
$$




$$
=\left[\int_{Y}\left(\varphi \circ F^{p}\right)(y) d \lambda(y)\right]^{\frac{1}{p}}\left[\int_{Y}\left(\varphi \circ G^{q}\right)(y) d \lambda(y)\right]^{\frac{1}{q}} .
$$

Using Theorem 2.1 we obtain

$$
\begin{gathered}
{\left[\int_{Y}\left(\varphi \circ F^{p}\right)(y) d \lambda(y)\right]^{\frac{1}{p}}\left[\int_{Y}\left(\varphi \circ G^{q}\right)(y) d \lambda(y)\right]^{\frac{1}{q}} \geq \varphi\left(\int_{Y} F(y) d \lambda(y) \int_{y} G(y) d \lambda(y)\right)} \\
=\varphi\left(\int_{Y}\left(\int_{X} f^{p}(x) \omega(x, y) d \mu(x)\right)^{\frac{1}{p}}\left(\int_{X} g^{q}(x) \omega(x, y) d \mu(x)\right)^{\frac{1}{q}} d \lambda(y)\right) \\
\geq\left[\varphi\left(\int_{Y} F^{p}(y) d \lambda(y)\right)\right]^{\frac{1}{p}}\left[\varphi\left(\int_{Y} G^{q}(y) d \lambda(y)\right)\right]^{\frac{1}{q}} \\
\left.=\left[\int_{Y}\left(\int_{X} f^{p}(x) \omega(x, y) d \mu(x) d \lambda(y)\right)\right)\right]^{\frac{1}{p}}\left[\varphi\left(\int_{Y}\left(\int_{X} g^{q}(x) \omega(x, y) d \mu(x) d \lambda(y)\right)\right)\right]^{\frac{1}{q}} \\
=\left[\varphi\left(\int_{X} f^{p}(x)\left(\int_{Y} \omega(x, y) d \lambda(y)\right) d \mu(x)\right)\right]^{\frac{1}{p}}\left[\varphi\left(\int_{X} g^{q}(x)\left(\int_{Y} \omega(x, y) d \lambda(y)\right) d \mu(x)\right)\right]^{\frac{1}{q}} \\
=\left[\varphi\left(\int_{X} f^{p}(x) d \mu(x)\right)\right]^{\frac{1}{p}}\left[\varphi\left(\int_{X} g^{q}(x) d \mu(x)\right)\right]^{\frac{1}{q}} \\
=\left[\int_{X} f g d \mu(x)\right) .
\end{gathered}
$$

Remark 2.5. Theorem 2.4 refines the result obtained by Hewitt and Stromberg on page 202 of [1] and also generalizes [3].

\section{References}

[1] E. Hewitt, K. Stromberg, "Real and Abstract Analysis", Springer-Verlag, New York, (1965)

[2] P. O. Olanipekun, A. A. Mogbademu, "A note on generalization of classical Jensen's inequality”, J.Math.Computer Sci. 13(2014), 68-70. 
[3] J. ROOIN, “A refinement of Jensen's inequality”, J. Inequal. Pure and Appl. Math., 6(2) Art. 38, (2005).

[4] W. RUDIN, "Real and Complex Analysis", $3^{\text {rd }}$ ed., McGraw-Hill, New York, (1974). 